\section{Fluid dynamics}

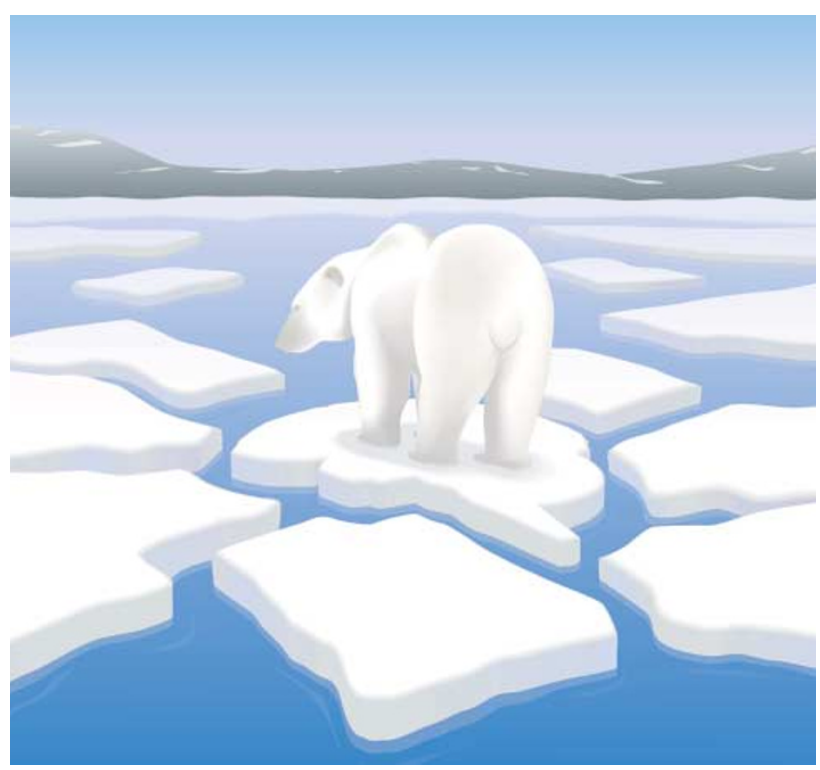

Epidemiological studies have indicated that cholesterol-lowering drugs, such as the statins, can reduce the risk of developing Alzheimer's disease (AD). Paradoxically, however, reducing cholesterol levels in the rodent brain seems to promote neurodegeneration. To try to resolve this apparent contradiction, AbadRodriguez and colleagues carried out a more direct investigation of the effects of neuronal membrane cholesterol levels on the production of the amyloid- $\beta(A \beta)$ peptide the main constituent of the amyloid plaques that form in the brains of patients with $\mathrm{AD}$.

Cleavage of amyloid precursor protein (APP) to generate $A \beta$ was previously thought to occur predominantly in cholesterol-rich microdomains of the neuronal membrane, known as rafts or detergent-resistant membrane (DRM) microdomains. However, the evidence for this model came largely from experiments that involved overexpression of amyloid precursor protein (APP) and the APP-cleaving secretase enzymes.

Abad-Rodriguez et al. showed that when APP and the $\beta$-secretase BACE1 were expressed at physiological levels in the membranes of human and rodent hippocampal neurons, APP was almost entirely excluded from the DRMs, whereas BACE1 was present in both DRM and non-DRM fractions. If the membrane fluidity was increased by reducing the cholesterol level, APP and BACE1 were co-localized more frequently, and this led to a rise in $A \beta$ production.

These findings contradict the idea that $\mathrm{A} \beta$ synthesis takes place in the DRM domains - in fact, the cholesterol in these domains seems to function as a barrier to the interaction between APP and BACE1. Loss of cholesterol from the membrane probably releases BACE1 from the DRMs, making it more likely to

\title{
Computer addict
}

It has been proposed that addictive drugs and reinforcement learning might influence the same neurophysiological pathways. In a recent paper in Science, Redish uses this hypothesis to generate a computational model of addiction.

One of the effects of many drugs of abuse is an increase in dopamine levels in the brain, which is thought to contribute to the addictive quality of these drugs. Natural rewards are also accompanied by an increase in dopamine, although with learning this increase shifts from the time of reward to the time of the cuing stimulus.

Reinforcement learning occurs as a result of an individual's interaction with the environment - that is, in response to experience rather than explicit teaching. This process can be modelled using temporal-difference reinforcement learning (TDRL), a reinforcement learning algorithm that relies on an error-reward signal. TDRL models aim to attain maximum future reward, and learn, by means of various calculations, to function accordingly. In these models, learning only occurs when the reward is incorrectly predicted. With correctly predicted reward, there is no error signal, and therefore no learning.

Using dopamine as the error-reward signal, the computational model established by Redish shows what happens when a positive signal, much like the dopamine surge that would accompany the use of a drug such as cocaine, is introduced neuropharmacologically rather than occurring as a result of an unexpected natural reward or cue stimulus. This overrides the reward predictions of the TDRL model and, because the positive signal does not relate to the other factors included in the model's calculations, the model is unable to predict such rewards. This means that the likelihood of the model selecting a pathway that would lead to drug reward depends on its number of experiences.

With learning, TDRL models usually achieve a stable response to natural rewards. This response depends on the time to and level of a reward and any discounting factors, which decrease the expected value of the reward. This sensitivity between natural reward and cost is called elasticity. In Redish's modified TDRL, the demand for drug reward increases disproportionately, so that although the process still shows some elasticity, it is inelastic when compared with natural reward. However, this does not necessarily mean that a drug reward would always be selected over a non-drug reward, as selection would depend on the size of the non-drug reward relative to that of the drug reward.

It is hoped that computational models of addiction such as this one will help us to understand the mechanisms and factors that are involved in addiction. Such models could be used to help explain and confirm observations, and to make further predictions that can be tested in the future. Sarah Archibald

\section{(i) References and links} ORIGINAL REFERENCE PAPER Redish, A. D. Addiction as a computational process gone awry. Science $\mathbf{3 0 6}$, 1944-1947 (2004) FURTHER READING Wise, R. A. Dopamine, learning and memory. Nature Rev. Neurosci. 5, 483-494 (2004)| Koob, G. F. \& Le Moal, M. Drug addiction, dysregulation of reward, and allostasis. Neuropsycopharmacology 24, 97-129 (2001) 
encounter APP in the more fluid regions of the membrane.

So, how can these observations be reconciled with the effects of statins in humans? As Abad-Rodriguez et al. point out, most of the commonly used statins are poor penetrators of the blood-brain barrier, so their benefits might derive from antiinflammatory or antioxidant properties rather than direct effects on brain neurons. Their new findings indicate that, rather than trying to reduce brain cholesterol, care should actually be taken to preserve the cholesterol balance in the membranes of brain neurons.

Heather Wood

\begin{abstract}
(9) References and links ORIGINAL RESEARCH PAPER AbadRodriguez, J. et al. Neuronal membrane cholesterol loss enhances amyloid peptide generation. J. Cell Biol. 167, 953-960 (2004) FURTHER READING Kaether, C. \& Haass, C. A lipid boundary separates APP and secretases and limits amyloid $\beta$-peptide generation. J. Cell Biol. 167, 809-812 (2004) | Citron, M. Strategies for disease modification in Alzheimer's disease. Nature Rev. Neurosci. 5, 677-685 (2004)
\end{abstract}

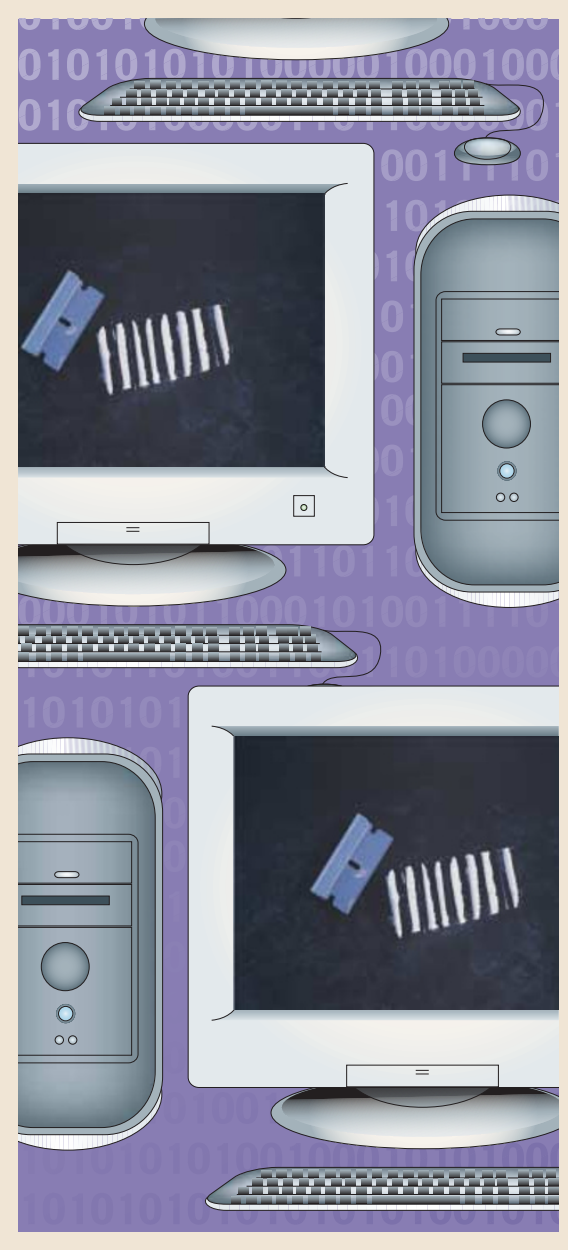

\section{SYNAPTIC PHYSIOLOGY}

\section{Fuel for plasticity}

Mitochondria are the energy factories of cells. Although it is generally thought that their activity adapts to cellular physiology, the functional significance of mitochondrial distribution in the cell is largely unknown. Reporting in Cell, $\mathrm{Li}$ and colleagues show that synaptic activity and mitochondrial distribution are reciprocally regulated.

Mitochondria are abundant in the cell bodies and large-diameter proximal dendrites of cultured hippocampal neurons, but are present at much lower densities in small-diameter and distal dendrites. There is a temporal correlation between increased mitochondrial incursion into dendritic protrusions and active synapse formation, which indicates that mitochondrial function might be crucial for the development of synapses.

Repetitive depolarization of cultured hippocampal neurons, which results in the formation of new dendritic spines and morphological changes to existing ones, also leads to an increase in the proportion of dendritic protrusions that contain mitochondria. Similarly, after local synaptic stimulation of neurons in organotypic hippocampal slices, mitochondria accumulate in the vicinity of active synapses and invade enlarged spines. This process can be blocked by an NMDA ( $N$-methyl-D-aspartate) receptor antagonist, which indicates that the increased mitochondrial presence is due to synaptic excitation.

To test whether mitochondria are required for synaptic development and maintenance, $\mathrm{Li}$ and colleagues perturbed mitochondrial distribution in clutured hippocampal neurons by overexpressing wild-type and dominant-negative mutant forms of dynamin-related protein 1 (DRP1) and optic atrophy 1 (OPA1). DRP1 and OPA1 are large GTPases of the dynamin family and are important regulators of the fission and fusion processes of mitochondria, respectively.

In neurons that were transfected with dominant-negative DRP1 (DRP1-K38A) or wild-type OPA1 - treatments that decreased the amount of mitochondria in dendrites - the density of dendritic spines was greatly reduced. By contrast, the density of dendritic spines increased almost two-fold in neurons that overexpressed wild-type DRP1. Creatine, a drug that enhances mitochondrial function, mimics the effect of DRP1 on synaptic density, indicating that the promoting effect of DRP1 on synapse formation might result from increased numbers of dendritic mitochondria. Consistent with this finding, both creatine and wild-type DRP1

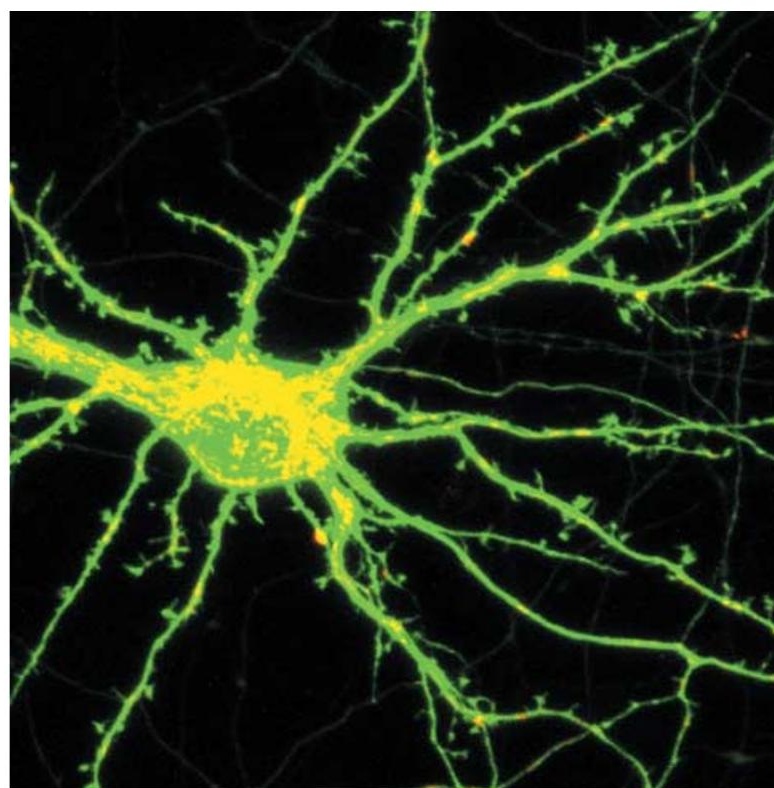

Mitochondria (stained with MitoDsRed) are seen as yellow in the cell body and dendrites of a neuron that has been transfected with green fluorescent protein. Image courtesy of Z. Li, Massachusetts Institute of Technology, USA.

enhance the synaptogenic response of neurons to repetitive stimulation.

What effect does global neuronal activity have on the balance of mitochondrial fission and fusion and the motility of dendritic mitochondria? Drugs that block action potentials, such as tetrodotoxin, were shown to increase the rate of mitochondrial movement and the ratio of mitochondrial fusion/fission in cultured hippocampal neurons, whereas depolarization of neurons by $\mathrm{KCl}$ produced opposing effects. Overexpression of DRP1-K38A blocked the change in mitochondrial motility that was induced by tetrodotoxin, which indicates that DRP1 is required for activity-dependent regulation of mitochondrial motility. In addition, DRP1-K38A increased the ratio of mitochondrial fusion/fission in neurons, whereas wild-type DRP1 decreased it.

This study shows a structural and functional interplay between dendritic mitochondria and synapses. As abnormal mitochondrial morphology and function have been associated with neurodegenerative diseases, the findings raise the possibility that the characteristic loss of synapses in these disorders might arise, in part, from mitochondrial dysfunction.

Jane Qiu

6) References and links ORIGINAL RESEARCH PAPER $L i, Z$, et $a$ l. The importance of dendritic mitochondria in the morphogenesis and plasticity of spines and synapses. Cell 119, 873-887 (2004)

FURTHER READING Birky, C. W. J. The inheritance of genes in mitochondria and chloroplasts: laws, mechanisms and models. Annu. Rev. Genet. 35, 125-148 (2001) WEB SITE

Sheng's laboratory:

http://www.hhmi.org/research/investigators/sheng.html 